\title{
Pancreatic Metastases from Tumors in the Urogenital Tract
}

\author{
Oliver Strobel Markus W. Büchler \\ Department of General, Visceral and Transplantation Surgery, Heidelberg University \\ Hospital, Heidelberg, Germany
}

\section{Key Words}

Pancreatic metastases · Urogenital tract · Surgical resection

\begin{abstract}
Background: Isolated pancreatic metastases or pancreatic metastases with limited extrapancreatic disease are uncommon and account for only $2-4 \%$ of resected malignant pancreatic lesions in surgical series. However, clear-cell renal cell carcinoma is the predominant primary tumor and accounts for more than $60 \%$ of cases with isolated pancreatic metastases. Pancreatectomy is the treatment of choice for most patients with isolated pancreatic metastases from renal cell cancer. Summary: This review provides an overview of clinical presentation and diagnosis as well as surgical management, including patient selection for surgery and surgical technique for pancreatic metastases of renal cell carcinoma. Key Message: Although there is no high-level evidence that surgical resection of pancreatic metastases improves survival, the survival results of several observational series and of systematic reviews are promising and support pancreatic resection as part of a multimodal treatment. The reported median survival and 5-year survival rates after pancreatic resection range from 6 to 10 years and from 55 to $75 \%$, respectively. Pancreatic resection is effective for local control. However, extrapancreatic progression frequently occurs. With the introduction of novel systemic therapy options such as tyrosine kinase inhibitors, the prognosis of metastatic renal cell carcinoma has improved, and this will affect the role of pancreatic resection for metastases. Practical Implications: Pancreatic resection for isolated renal cell carcinoma is safe and effective, may confer a survival benefit and should, therefore, be considered in patients for whom no contraindication for surgery exists.

(C) 2015 S. Karger AG, Basel
\end{abstract}




\section{The Pancreas as a Site of Metastases}

Resection has become an established part of the standard therapy for liver and lung metastases of colorectal cancer and of several other primary tumors [1, 2]. The value and benefit of surgical resection for metastatic disease largely depends on the biological behavior of the primary tumor and the availability of effective systemic treatments. Unlike the liver and lung, the pancreas is an uncommon site of metastases [3]. In an autopsy series 3-12\% of patients with diffuse systemic disease have pancreatic metastases [4]. Metastases account for only about $2-4 \%$ of malignant lesions in the pancreas in a series of surgical resection [3]. However, this number may increase up to $40 \%$ in patients with a pancreatic mass and a history of malignant disease, especially renal cell carcinoma (RCC). The literature is dominated by multiple case reports and small case series and a few larger observational studies of surgical resection for pancreatic metastases. Many of these reports are heterogeneous and include the following: (1) metastases of different primary tumors, (2) isolated pancreatic metastases and pancreatic metastases in the context of limited (resectable) extrapancreatic disease and (3) synchronous and metachronous pancreatic metastases. Clear-cell RCC is by far the most common primary tumor for isolated pancreatic metastases and dominates the surgical series, accounting for more than $60 \%$ of cases $[5,6]$. All other primary tumors are much less frequent, and other primary tumors from the urogenital tract are very rarely the origin of isolated pancreatic metastasis (table 1).

In the following the management of pancreatic metastases from RCC will be discussed with a focus on the role of surgical therapy, including clinical presentation, diagnostic workup, patient selection, and resection technique.

\section{Clinical Presentation and Diagnostic Workup}

With respect to the clinical presentation, patients with pancreatic metastases can be divided into patients that present with symptoms and patients in whom the metastases are detected by cross-sectional imaging during a routine oncological follow-up examination or as an incidental finding of imaging for other reasons. Therefore, the clinical presentation largely depends on the existence of and compliance with routine oncological surveillance programs as well as the biology of the primary tumor entity. Isolated pancreatic metastases for RCC typically become evident as a metachronous disease with a long interval of 10 and more years after primary tumor resection even of small RCC $[6,7]$. Therefore, many of the affected patients may already have been discharged from surveillance and present due to symptoms. In studies that include patients from previous decades, $60-90 \%$ of patients presented due to symptoms [7, 8], whereas in our own series only $18 \%$ of metastases were diagnosed due to symptoms and $82 \%$ were detected during oncological follow-up [6]. This shift is most likely due to increasing adherence to standardized follow-up programs and broader use of crosssectional imaging techniques. In a recent systematic review of 399 patients ( 250 with RCC) the mean age of patients with pancreatic metastases was 61.7 years, with $42 \%$ female and $40 \%$ symptomatic at presentation [9]. Dependent of the location of the metastases in the pancreas, the most frequent complaints of symptomatic patients are upper abdominal pain, obstructive jaundice and gastrointestinal bleeding. Symptomatic patients tend to have bigger lesions than asymptomatic patients, and patients with symptoms appear to have a worse prognosis [8]. These findings suggest that symptoms will eventually develop as local complications during the progression of pancreatic metastases and point to an indication for surgical resection not only in curative intention but also for palliation. 


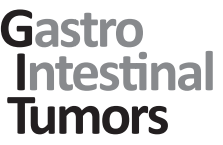

Table 1. Pathological diagnosis in patients with pancreatic resections for metastases

\begin{tabular}{l|l}
\hline Gastrointest Tumors 2015;2:75-82 \\
\hline DOI: 10.1159/000431045 & $\begin{array}{l}\text { C 2015 S. Karger AG, Basel } \\
\text { www.karger.com/gat }\end{array}$ \\
\hline
\end{tabular}

Strobel and Büchler: Pancreatic Metastases from Tumors in the Urogenital Tract

\begin{tabular}{lrr}
\hline Primary tumor type & $\mathrm{n}$ & $\%$ \\
\hline RCC & 181 & 63.1 \\
Colorectal cancer & 22 & 7.7 \\
Sarcoma & 17 & 5.9 \\
Melanoma & 13 & 4.5 \\
Gastric cancer & 10 & 3.5 \\
Lung cancer & 9 & 3.1 \\
Gall bladder cancer & 8 & 2.9 \\
Breast cancer & 6 & 2.1 \\
Ovarian cancer & 5 & 1.7 \\
Gastrointestinal stromal tumor & 2 & 0.7 \\
Esophageal cancer & 2 & 0.7 \\
Mesenteric fibromatosis & 2 & 0.7 \\
Schwannoma & 2 & 0.7 \\
Merkel cell carcinoma & 1 & 0.3 \\
Seminoma & 1 & 0.3 \\
Teratocarcinoma & 1 & 0.3 \\
Hemangiopericytoma & 1 & 0.3 \\
Urinary bladder cancer & 1 & 0.3 \\
Carcinoid & 1 & 0.3 \\
Nonpancreatic endocrine tumor & 1 & 0.3 \\
Hepatocellular carcinoma & 1 & 0.3 \\
\hline
\end{tabular}

Based on combined data from Reddy et al. [5] and Strobel et al. [6] and modified from Strobel et al. [3]. Primary tumors in the urogenital tract are printed in italics.

The preferred imaging for staging and surveillance is contrast-enhanced CT followed by MRI. Pancreatic metastasis of RCC typically present as hypervascularized, contrast-enhancing lesions that are best visible in the arterial phase [10]. A typical CT finding is demonstrated in figure 1 . Another feature of metastases is their multifocality within the pancreas $(40 \%$ in pathological workup in our own series [6]). In patients with a history of RCC such lesions are highly suspicious for pancreatic metastases. A biopsy is usually not required. In contrast, the importance of a state-of-the-art restaging is highlighted by the high frequency of additional extrapancreatic lesions in patients with pancreatic metastases. A thorough workup may include a whole-body CT scan. If additional extrapancreatic disease is suspected, a PET scan is recommended to identify patients with diffuse spread who will not benefit from surgery.

While disseminated metastatic disease is a contraindication for surgery, the option of surgical resection should be evaluated in isolated pancreatic metastases or pancreatic metastases with limited extrapancreatic disease.

\section{Surgery for Pancreatic Metastases of RCC}

Within the last decade the indications for pancreatic surgery have been significantly extended, mainly due to improvements in surgical technique, resulting in considerably decreased morbidity and mortality of pancreatic resections [11]. At the same time the prognosis of many cancers has improved even in the metastatic situation with the development of more effective drugs and targeted therapies. While some years ago the literature on pancreatic resections for metastases consisted only of case reports and small series that were certainly subject to publication bias, there are meanwhile several larger (10-44 cases of RCC 


\section{Tumors}

Strobel and Büchler: Pancreatic Metastases from Tumors in the Urogenital Tract

Fig. 1. Typical radiological features of a pancreatic metastasis from RCC. CT scan of a 52-yearold female patient who presented with obstructive jaundice 10.8 years after left nephrectomy for clear-cell RCC pT1, pN0, G2, R0. Preoperative contrast-enhanced CT scan. a Arterial phase in axial orientation. b Venous phase in axial orientation. c Venous phase in coronal orientation. The hypervascular lesion in the pancreatic head (arrows) exhibits intense contrast enhancement especially in the arterial phase and is less evident in the venous phase. The lesion results in compression of the common bile duct with subsequent cholestasis (asterisks).
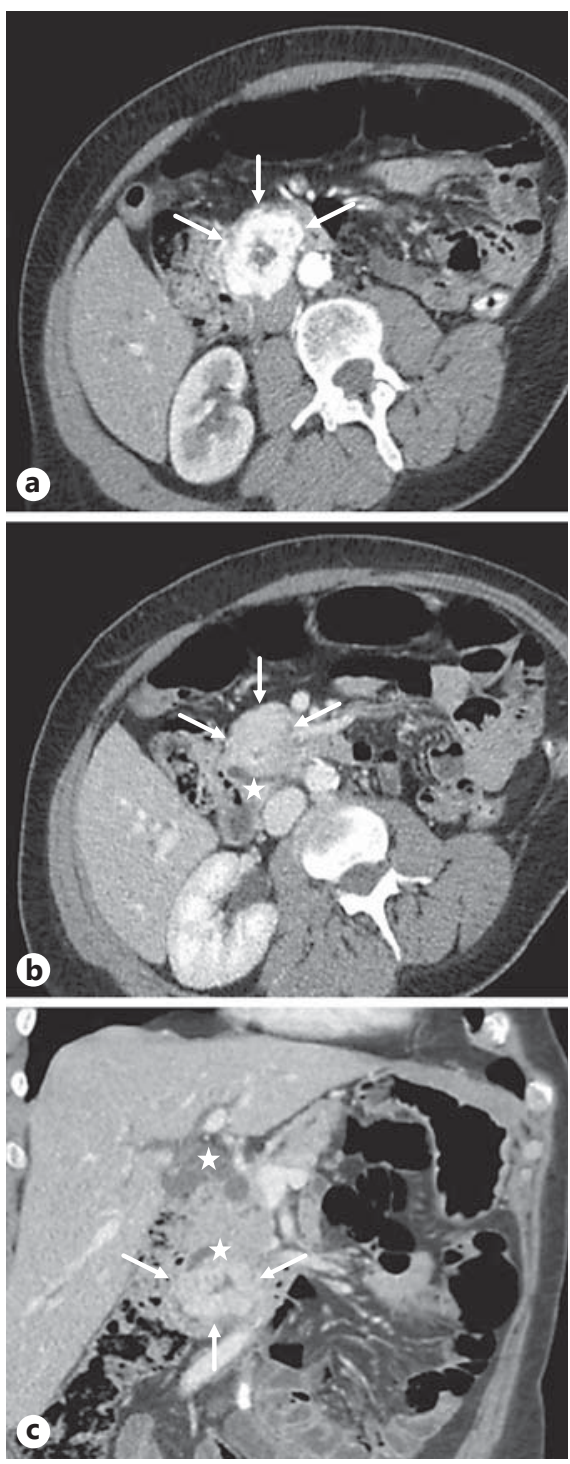

metastases) retrospective series [5, 6, 12-25] and even systematic reviews [7, 8, 26-28] on surgical resection for pancreatic metastases and RCC (table 2). However, there are still no prospective studies or randomized controlled trials on this topic. Overall, the recent series and the systematic reviews suggest that resection for pancreatic metastases is safe, effective and associated with favorable long-term outcome. This is best documented for RCC, which is by far the predominant primary tumor in pancreatic metastases (table 1). Based on these recent data, pancreatic resection can be recommended as part of an interdisciplinary treatment for RCC metastasis to the pancreas.

\section{Safety of Resection for Pancreatic Metastases}

In a recent systematic review including 18 series with more than 10 cases and a total of 399 patients a mortality rate of $2.21 \%$ and a morbidity rate of $39.85 \%$ (including minor complications) were reported for resection of isolated pancreatic metastases [9]. Resections for metastatic disease may be more difficult than those for primary pancreatic tumors due to the necessity of adhesiolysis after previous surgery in around $80 \%$ of cases and the 


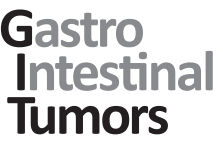

Table 2. Outcome after pancreatic resection for RCC metastases

\begin{tabular}{l|l}
\hline Gastrointest Tumors 2015;2:75-82 \\
\hline DOI: 10.1159/000431045 & $\begin{array}{l}\text { @ 2015 S. Karger AG, Basel } \\
\text { www.karger.com/gat }\end{array}$ \\
\hline
\end{tabular}

\begin{tabular}{llll}
\hline Study & $\mathrm{n}$ & Median survival & 5-year survival \\
\hline Series & & & \\
Yuasa et al. [24], 2015 & 15 & not reached & $72 \%$ \\
Santoni et al. [18], 2015 & 44 & 8.6 years & $\mathrm{NR}$ \\
Tosoian et al. [20], 2014 & 42 & 5.5 years & $52 \%$ \\
Untch and Allen [21], 2014 & 27 & 8 years & $\mathrm{NR}$ \\
Facy et al. [13], 2013 & 13 & NR & $75 \%$ \\
Yazbek and Gayet [23], 2012 & 11 & 6.5 years & $\mathrm{NR}$ \\
Konstantinidis et al. [16], & & & \\
$\quad$ 2010 & 20 & 8.7 years & $61 \%$ \\
Strobel et al. [6], 2009 & 29 & not reached & $67 \%$ \\
Volk et al. [22], 2009 & 14 & 6.3 years & $\mathrm{NR}$ \\
Reddy et al. [5], 2008 & 21 & 4.8 years & $45 \%$ \\
Zerbi et al. [25], 2008 & 23 & not reached & $88 \%$ \\
Bassi et al. [12], 2003 & 17 & not reached & $53 \%$ \\
Law et al. [17], 2003 & 14 & not reached & $75 \%$ \\
Hiotis et al. [15], 2002 & 10 & 4.8 years & $\mathrm{NR}$ \\
Sohn et al. [19], 2001 & 10 & not reached & $75 \%$ \\
Ghavamian et al. [14], 2000 & 11 & 10 years & $81 \%$ \\
\hline Reviews & & & \\
Adler et al. [26], 2014 & 250 & 6 years & $70.4 \%$ \\
Sweeney et al. [28], 2010 & 135 & 5.8 years & $65 \%$ \\
Reddy and Wolfgang [27], & & & \\
$\quad$ 2009 & 112 & $8.8 \%$ & $66 \%$ \\
Tanis et al. [8], 2009 & 311 & NR & $72.6 \%$ \\
Sellner et al. [7], 2006 & 139 & 10 years & $72 \%$ \\
\hline
\end{tabular}

Series: only series with 10 or more cases of RCC that report either median or 5-year survival or both. NR = Not reported.

necessity of multivisceral resections. In our own series, which included $41 \%$ of multivisceral resections, we reported a mortality rate of $4.4 \%$ and a morbidity rate of $33 \%$ [6]. These findings are comparable to the number reported for primary pancreatic malignancies and show that resection for pancreatic metastases can be performed safely by specialized surgeons.

\section{Oncological Outcome after Resection for Pancreatic RCC Metastases}

The oncological long-term results of the largest series and of several systematic reviews of pancreatic resection for RCC metastases are summarized in table 2 and show a favorable outcome. Overall, the reported median survival varies between 5 and 10 years and the reported 5-year survival rates vary between 44 and $88 \%$. This heterogeneity has several reasons, including the following: (1) some series include only isolated pancreatic metastases, while others also include patients with resectable extrapancreatic disease, (2) the follow-up times vary between studies and (3) the early systematic reviews included case reports, and small series and are more subject to publication bias. The recent systematic review by Adler et al. [9] probably provides the most solid survival data and reports a median survival of 71.7 months and a 5-year survival rate of $70.4 \%$ after resection of pancreatic metastases of RCC. In series including several primary tumors RCC histology was consistently associated with longer survival compared to other primary tumors [5, 6, 15, 29].

Although $57 \%$ of cases in our own series had additionally extrapancreatic metastatic disease, we observed an overall 5-year survival rate of $56.8 \%$ and that of $67.3 \%$ after resection 
of RCC metastases; the 5-year survival rate after resection of isolated pancreatic metastases was $75 \%[6]$.

\section{Efficacy of Resection for Pancreatic Metastases}

With the lack of controlled trials comparing resection to conservative management, the true effect of pancreatic resection on survival cannot be formally proven. Three reports observed longer survival in patients after resection compared to noncontrolled groups with unresectable disease, but this may be explained by selection $[7,8,25]$. The value of metastasectomy also depends on the efficacy of the available systemic treatment. The development of novel drugs such as tyrosine kinase inhibitors has improved survival of metastasized RCC, and this may impact the value of surgical resection. A recent noncontrolled study retrospectively compared management by surgical resection in 44 patients to conservative treatment with tyrosine kinase inhibitors in 59 patients and observed a median overall survival of 103 months versus 86 months, respectively $(p=0.201)$ [18]. By multivariate analysis surgical resection was not independently associated with survival. However, $18 \%$ of the resected patients remained disease free. Future studies have to show the value of surgery for pancreatic RCC metastases in the era of novel and more effective systemic treatments. As a cure can only be achieved with surgical resection, it can be expected that pancreatic resections for RCC metastases will be increasingly performed and will be established as part of the multimodal standard treatment even in the absence of randomized controlled trials, as was the case with hepatic and pulmonary resections for colorectal cancer metastasis.

Apart from the long overall survival, another point supporting resection for pancreatic metastases is the good local tumor control. In our series the local tumor control after 5 years was $87 \%$, while the 5 -year disease-free survival rate was only $33 \%$ because of distant metastases [6]. On the one hand, this underlines the importance of multimodal therapy, including systemic treatment, in these patients. On the other hand, these data show that pancreatic resection is highly effective for local control, including the control of the symptoms associated with pancreatic metastasis such as pain, cholestasis and gastrointestinal bleeding.

\section{Patient Selection for Surgery}

Decision making and selection of patients for surgery has to be performed individually based on several parameters that should include patient age, comorbidity and resulting perioperative risk, the interval between primary tumor resection and pancreatic metastases, and the pattern of metastatic spread. Ideally, the decision process should involve both the medical oncologist and experienced pancreatic surgeons and does not differ much from decision making for resection of other metastatic sites such as the liver and lung.

\section{History of the Disease}

A prolonged interval (e.g. $>36$ months) between the primary tumor and the occurrence of the pancreatic metastasis is associated with improved survival. Of importance, a previous recurrence appears to affect disease-free but not overall survival after pancreatic metastasectomy [6].

\section{Pattern of Recurrent Disease}

As one may expect, the presence of extrapancreatic disease is associated with shorter disease-free and overall survival [6]. This further highlights the importance of a thorough diagnostic workup to rule out local or extrapancreatic systemic recurrence of the primary 
tumor, as discussed above. However, the survival results show that additional extrapancreatic disease should not preclude a patient from resection if the pattern is not multifocal/ diffuse and if a complete resection can be expected.

\section{Local Resectability}

In contrast to pancreatic ductal adenocarcinoma, pancreatic metastases are in most cases easily resectable from a technical point of view. However, a total pancreatectomy is often necessary for multifocal pancreatic metastases. Based on preoperative imaging, this aspect has to be taken into account, and the consequence of brittle diabetes has to be thoroughly discussed with the patients. With appropriate management total pancreatectomy is associated with a good quality of life and does not negatively affect survival [30].

In the recent study by Santoni et al. [31], the Memorial Sloan Kettering Cancer Center prognostic criteria for advanced RCC are an independent predictor of survival in patients with pancreatic RCC metastases and may, therefore, be useful for patient selection [18].

\section{Technique of Resection for Pancreatic RCC Metastases}

There are no studies comparing different techniques of pancreatic resection for metastases. However, most larger studies have described radical resections, and in one study a high rate of pancreatic recurrences was observed after limited resections [12]. In our series we performed standard partial resections but avoided total pancreatectomy whenever possible and observed only 2 pancreatic recurrences after 30 partial pancreatectomies (6.7\%) [6]. As pancreatic metastases are frequently multifocal, a thorough exploration of the pancreatic remnant by palpation and intraoperative ultrasound is mandatory whenever a partial pancreatectomy is performed. As the two largest series showed that pancreatic metastases of RCC are associated with peripancreatic lymph node metastases in $26-30 \%$ of cases $[5,6]$, a standard peripancreatic lymphadenectomy should be performed during pancreatic resection for metastases. Overall, standard partial pancreatectomy combined with standard lymphadenectomy rather than limited local resection is recommended for the treatment of pancreatic RCC metastases. If complete tumor resection can be achieved by total pancreatectomy and multivisceral resections these procedures should be performed.

\section{Conclusion}

Metastases of RCC account for the vast majority of pancreatic metastases. Isolated pancreatic metastases of RCC typically appear with a long interval of 10 years and more after resection of the primary tumor. This should be taken into account for the design of surveillance after the resection of RCC. Pancreatic RCC metastases have typical imaging features and present as hypervascularized, contrast-enhancing lesions that are best visible by CT scan in the arterial phase. Surgical resection of isolated RCC metastases is associated with favorable survival and should always be considered as part of a multimodal treatment in patients who are fit for surgery.

\section{Disclosure Statement}

The authors have nothing to disclose. 


\section{References}

1 Fong Y, Fortner J, Sun RL, Brennan MF, Blumgart LH: Clinical score for predicting recurrence after hepatic resection for metastatic colorectal cancer: analysis of 1,001 consecutive cases. Ann Surg 1999;230:309-318.

-2 Quiros RM, Scott WJ: Surgical treatment of metastatic disease to the lung. Semin Oncol 2008;35:134-146.

3 Strobel 0, Büchler M, Werner J: The pancreas as a site of metastatic cancer; in Jarnagin W (ed): Blumgart's Surgery of the Liver, Bilary Tract, and Pancreas, ed 5. Philadelphia, Elsevier Saunders, 2012, pp 930-933.

4 Showalter SL, Hager E, Yeo CJ: Metastatic disease to the pancreas and spleen. Semin Oncol 2008;35:160-171.

5 Reddy S, Edil BH, Cameron JL, Pawlik TM, Herman JM, Gilson MM, et al: Pancreatic resection of isolated metastases from nonpancreatic primary cancers. Ann Surg Oncol 2008;15:3199-3206.

-6 Strobel O, Hackert T, Hartwig W, Bergmann F, Hinz U, Wente MN, et al: Survival data justifies resection for pancreatic metastases. Ann Surg Oncol 2009;16:3340-3349.

7 Sellner F, Tykalsky N, De Santis M, Pont J, Klimpfinger M: Solitary and multiple isolated metastases of clear cell renal carcinoma to the pancreas: an indication for pancreatic surgery. Ann Surg Oncol 2006;13:75-85.

-8 Tanis PJ, van der Gaag NA, Busch OR, van Gulik TM, Gouma DJ: Systematic review of pancreatic surgery for metastatic renal cell carcinoma. Br J Surg 2009;96:579-592.

$\checkmark 9$ Adam R, de Haas RJ, Wicherts DA, Aloia TA, Delvart V, Azoulay D, et al: Is hepatic resection justified after chemotherapy in patients with colorectal liver metastases and lymph node involvement? J Clin Oncol 2008; 26:3672-3680.

10 Hoshino Y, Shinozaki H, Kimura Y, Masugi Y, Ito H, Terauchi T, et al: Pancreatic metastases from renal cell carcinoma: a case report and literature review of the clinical and radiological characteristics. World J Surg Oncol 2013;11:289.

11 Hartwig W, Werner J, Jäger D, Debus J, Büchler MW: Improvement of surgical results for pancreatic cancer. Lancet Oncol 2013;14:e476-e485.

12 Bassi C, Butturini G, Falconi M, Sargenti M, Mantovani W, Pederzoli P: High recurrence rate after atypical resection for pancreatic metastases from renal cell carcinoma. Br J Surg 2003;90:555-559.

-13 Facy 0, Angot C, Guiu B, Al Samman S, Matte A, Rat P, et al: Interest of intraoperative ultrasonography during pancreatectomy for metastatic renal cell carcinoma. Clin Res Hepatol Gastroenterol 2013;37:530-534.

14 Ghavamian R, Klein KA, Stephens DH, Welch TJ, LeRoy AJ, Richardson RL, et al: Renal cell carcinoma metastatic to the pancreas: clinical and radiological features. Mayo Clin Proc 2000;75:581-585.

15 Hiotis SP, Klimstra DS, Conlon KC, Brennan MF: Results after pancreatic resection for metastatic lesions. Ann Surg Oncol 2002;9:675-679.

16 Konstantinidis IT, Dursun A, Zheng H, Wargo JA, Thayer SP, Fernandez-del Castillo C, et al: Metastatic tumors in the pancreas in the modern era. J Am Coll Surg 2010;211:749-753.

$\checkmark 17$ Law CH, Wei AC, Hanna SS, Al Zahrani M, Taylor BR, Greig PD, et al: Pancreatic resection for metastatic renal cell carcinoma: presentation, treatment, and outcome. Ann Surg Oncol 2003;10:922-926.

-18 Santoni M, Conti A, Partelli S, Porta C, Sternberg CN, Procopio G, et al: Surgical resection does not improve survival in patients with renal metastases to the pancreas in the era of tyrosine kinase inhibitors. Ann Surg Oncol 2015;22:2094-2100.

19 Sohn TA, Yeo CJ, Cameron JL, Nakeeb A, Lillemoe KD: Renal cell carcinoma metastatic to the pancreas: results of surgical management. J Gastrointest Surg 2001;5:346-351.

-20 Tosoian JJ, Cameron JL, Allaf ME, Hruban RH, Nahime CB, Pawlik TM, et al: Resection of isolated renal cell carcinoma metastases of the pancreas: outcomes from the Johns Hopkins Hospital. J Gastrointest Surg 2014;18:542-548.

-21 Untch BR, Allen PJ: Pancreatic metastasectomy: the Memorial Sloan-Kettering experience and a review of the literature. J Surg Oncol 2014;109:28-30.

22 Volk A, Kersting S, Konopke R, Dobrowolski F, Franzen S, Ockert D, et al: Surgical therapy of intrapancreatic metastasis from renal cell carcinoma. Pancreatology 2009;9:392-397.

23 Yazbek T, Gayet B: The place of enucleation and enucleo-resection in the treatment of pancreatic metastasis of renal cell carcinoma. JOP 2012;13:433-438.

-24 Yuasa T, Inoshita N, Saiura A, Yamamoto S, Urakami S, Masuda H, et al: Clinical outcome of patients with pancreatic metastases from renal cell cancer. BMC Cancer 2015;15:46.

25 Zerbi A, Ortolano E, Balzano G, Borri A, Beneduce AA, Di Carlo V: Pancreatic metastasis from renal cell carcinoma: which patients benefit from surgical resection? Ann Surg Oncol 2008;15:1161-1168.

-26 Adler H, Redmond CE, Heneghan HM, Swan N, Maguire D, Traynor O, et al: Pancreatectomy for metastatic disease: a systematic review. Eur J Surg Oncol 2014;40:379-386.

27 Reddy S, Wolfgang CL: The role of surgery in the management of isolated metastases to the pancreas. Lancet Oncol 2009;10:287-293.

28 Sweeney AD, Wu MF, Hilsenbeck SG, Brunicardi FC, Fisher WE: Value of pancreatic resection for cancer metastatic to the pancreas. J Surg Res 2010;160:268-276.

29 Eidt S, Jergas M, Schmidt R, Siedek M: Metastasis to the pancreas - an indication for pancreatic resection? Langenbecks Arch Surg 2007;392:539-542.

-30 Hartwig W, Gluth A, Hinz U, Bergmann F, Spronk PE, Hackert T, et al: Total pancreatectomy for primary pancreatic neoplasms: renaissance of an unpopular operation. Ann Surg 2015;261:537-546.

-31 Motzer RJ, Mazumdar M, Bacik J, Berg W, Amsterdam A, Ferrara J: Survival and prognostic stratification of 670 patients with advanced renal cell carcinoma. J Clin Oncol 1999;17:2530-2540. 